\title{
Planejamento do controle estatístico de processos com baixa fração não conforme restrito a amostras pequenas
}

\author{
Adriana Leiras \\ Eugenio Kahn Epprecht \\ PUC-Rio \\ Antonio Fernando Branco Costa \\ UNESP
}

\begin{abstract}
Resumo
É apresentado o planejamento não convencional de gráficos de $n p$ para o monitoramento de processos caracterizados por uma fração não conforme muito pequena e cujo procedimento de inspeção é custoso ou demorado, tornando proibitivo o exame de grandes amostras. Sob tais circunstâncias, a base para o planejamento do gráfico de controle é o "trade-off' entre a freqüência de alarmes falsos e a rapidez com que o gráfico sinaliza deteriorações no processo, já que nestes casos (dependendo do tamanho de amostra) o gráfico de np convencional com limites "de três sigma" oferece pouca proteção contra alarmes falsos ou é lento para detectar perturbações no processo. Este estudo foi motivado pelo caso real de um processo de empacotamento de cadernos de jornais. 0 número nominal de cadernos no pacote depende da espessura do caderno, e pacotes com um número de cadernos diferente do nominal são considerados não conformes.
\end{abstract}

Palavras-chave

Gráfico de $n p$, controle estatístico de processos, controle por atributos, planejamento de gráficos de controle.

\section{Design of the statistical control of low fraction nonconforming processes restricted to small samples}

\begin{abstract}
We present the non-conventional design of $\mathrm{np}$ charts used for the surveillance of processes characterized by a very low fraction nonconforming, with a costly and/or time-consuming inspection procedure, which makes the inspection of large samples prohibitive. Under these circumstances, the trade-off between the false-alarm rate and the speed with which the chart signals process deteriorations is the basis to design the control chart, once (depending on the sample size] the conventional np chart with "three-sigma" limits offers little protection against false alarms or is slow in detecting process disturbances. The study was motivated by the real case of a newspapers packaging process. The nominal number of newspapers in the package depends on the newspaper's breadth, and packages with a number of newspapers different from nominal are considered nonconforming.
\end{abstract}

Key words

np charts, statistical process control, control by attributes, design of control charts. 


\section{INTRODUĈ̣̃O}

Os gráficos de controle surgiram em 1924, quando Shewhart, então funcionário da Bell Laboratories, publicou relatório técnico visando divulgar os fundamentos de uma técnica estatística destinada ao controle de processos. No início, como era de se esperar, poucos acreditaram no potencial desta nova técnica. Pouco a pouco, no entanto, o gráfico de controle foi ganhando fama, e seu uso como ferramenta de monitoramento passou a ser obrigatório, em um número infindável de processos industriais. Já são vários os artigos em português que tratam do tema; por exemplo Costa (1998), Epprecht e Santos (1998), Mingoti e Fidelis, (2001), Michel e Fogliatto (2002) e Turnes et al. (2004). O gráfico de controle possui uma linha média e limites de ação que se distanciam desta por $k$ desvios-padrão da estatística amostral registrada no gráfico. Por exemplo, no gráfico de $\bar{X}$, os limites são situados a $\pm k \sigma_{0} / \sqrt{n}$ de $\mu_{0}$, sendo $\mu_{0}$ e $\sigma_{0}$, respectivamente, a média e o desvio-padrão em controle do processo, $n$ o tamanho de amostra, e $\sigma_{0} / \sqrt{n}$, o desviopadrão de $\bar{X}$. Um valor tradicional para o coeficiente $k$, recomendado por Shewhart e por outros autores (e.g. Wheeler, 1995) é $k=3$. Limites de controle calculados com $k=3$ são conhecidos como "limites de três sigma". Se a distribuição de $\bar{X}$ for normal e a média do processo estiver ajustada em seu valor alvo $\mu_{0}$, então, para cada amostra, haverá sempre uma probabilidade de 0,0027 de a estatística amostral $\bar{X}$ não pertencer ao intervalo delimitado pelos limites de ação do gráfico, ou seja, $\mu_{0} \pm 3 \sigma_{0} / \sqrt{\mathrm{n}}$, gerando portanto um sinal (ver COSTA et al., 2005). Em outras palavras, o risco de o gráfico de controle sinalizar indevidamente um desajuste na média do processo é de 0,0027 . Tal sinal é chamado de alarme falso. A incidência de alarmes falsos deve ser esporádica para que o operador não perca a confiança no dispositivo estatístico de monitoramento. Por outro lado, se a média do processo assumir um valor diferente de $\mu_{0}$, é importante que o gráfico de controle sinalize tal condição o quanto antes; neste caso, tem-se um alarme verdadeiro. Valores adequados para os limites de controle são importantes para limitar a probabilidade de alarme falso e garantir a rapidez do gráfico em emitir alarmes verdadeiros. O valor 3 para o parâmetro $k$ é um valor robusto: Wheeler (op. cit., cap. 5) mostra que as áreas das caudas de praticamente qualquer distribuição de probabilidades para além de três desvios-padrão de distância da média correspondem a uma probabilidade muito pequena. Assim, gráficos de controle com "limites de três sigma" geram poucos alarmes falsos, mesmo para os casos em que a estatística utilizada no monitoramento possui uma distribuição não-normal e assimétrica: gráficos de $R$, de $n p$, de $c$, por exemplo. Evidentemente, nesses casos, as probabilidades de alarme falso serão diferentes do valor nominal 0,0027 mas ainda serão pequenas (COSTA et al., 2005, apresentam uma análise detalhada dessas probabilidades para vários gráficos) e, pelo Teorema Central do Limite, independentemente da distribuição da característica de qualidade de interesse, quanto maior o tamanho da amostra, mais a distribuição da estatística amostral se aproximará da distribuição normal, de modo que a probabilidade de alarme falso tenderá para o valor 0,0027 .

No caso do gráfico de $n p$, que é o foco deste artigo, a estatística amostral é o número de defeituosos na amostra, que se supõe seguir uma distribuição binomial; assim, o Limite Superior de Controle ( $L S C$ ) e o Limite Inferior de Controle (LIC) de três sigma são dados por:

$$
L S C=n p_{0}+3 \sqrt{n p_{0}\left(1-p_{0}\right)}
$$

$$
L I C=n p_{0}-3 \sqrt{n p_{0}\left(1-p_{0}\right)}
$$

onde $p_{0}$ é o valor (conhecido, assumido, "alvo" ou estimado) da fração defeituosa média do processo no estado de controle estatístico. Se o valor de $L I C$ calculado resultar negativo, usa-se $L I C=0$, uma vez que o número de unidades defeituosas não pode ser negativo. $L I C=0$ significa, na verdade, a ausência de um limite inferior de controle (nessa situação, o gráfico de controle fica incapaz de sinalizar reduções na fração defeituosa média do processo, isto é, melhorias no mesmo). Isso ocorre para valores de $p_{0}$ pequenos e amostras não muito grandes. Por exemplo, $\operatorname{com} p_{0}=0,01$, a regra de "três sigma" requer amostras grandes, com mais de 891 unidades, para que $L I C>0$.

Se o tamanho de amostra for "grande", a distribuição da estatística amostral aproximar-se-á da distribuição normal e a probabilidade de alarme falso tenderá para o valor 0,0027 . O tamanho que pode ser considerado "grande" varia com o valor de $p_{0}$, pois a qualidade da aproximação normal para a distribuição binomial $(n, p)$ depende do produto $n p$. Para valores de $p_{0}$ típicos, que em geral são bem menores que 0,05 , para manter o risco de alarme falso em níveis próximos a 0,0027 são necessários tamanhos de amostra da ordem de centenas de unidades.

Porém, tais tamanhos de amostra nem sempre são possíveis: este trabalho foi originado pelo caso real de um processo que produz poucos itens não conformes $\left(p_{0}\right.$ pequeno), porém cuja inspeção é custosa ou demorada, tornando proibitiva a tomada de amostras grandes. O que fazer, nessa situação? Como será visto, nestes casos em que o valor de $n p_{0}$ é pequeno, o procedimento usual para planejar um gráfico de controle (escolher primeiro um tamanho de amostra e, com base nele, calcular os limites "de três sigma") é inaplicável, por produzir gráficos com riscos de alarme falso inaceitavelmente altos e/ou que são demasiadamente lentos na sinalização de alterações no processo. Enfatizando, não 
são apenas os limites de três sigma que não se aplicam: o próprio tamanho de amostra não pode ser objeto de uma decisão independente e anterior à determinação dos limites (no caso, do limite superior, único) de controle: a escolha de ambos, tamanho de amostra e limite superior de controle, deve ser conjunta, com base nos riscos de alarme falso e na rapidez de sinalização de alterações no processo. O objetivo deste trabalho é propor um procedimento para essa escolha conjunta.

O caso real que deu origem a este artigo é o do empacotamento de cadernos de jornais no parque gráfico de um grande jornal nacional. A característica de qualidade controlada é o número de cadernos no pacote (se este for diferente do número especificado, o pacote é não conforme). A fração não conforme em controle é muito baixa: $0,5 \%$. O procedimento de inspeção é a contagem manual de cadernos em um pacote. A lentidão inerente a esse procedimento, aliada ao fato de que a alta taxa de produção demanda amostragens freqüentes, impossibilita o exame de amostras grandes $(n=20$ já seria um tamanho impraticável). Com essas restrições, os limites "de três sigma" levam a taxas de alarme falso proibitivas, tais como um alarme falso a cada 40, 20 ou mesmo 14 amostras.

O procedimento proposto para o planejamento do controle estatístico de processos em tais condições é descrito na próxima seção, e a seguir, a título de ilustração, é detalhada a sua aplicação ao caso real de empacotamento de cadernos de jornais.

\section{PROCEDIMENTO PROPOSTO}

O procedimento consiste em examinar o leque de alternativas viáveis para o par de parâmetros $(n, L S C)$, analisando o desempenho previsto de cada uma delas, de modo a selecionar a melhor opção. Alternativas viáveis são os pares $(n, L S C)$ tais que $n$ esteja dentro da faixa de tamanhos de amostra possíveis na situação prática considerada, e tais que a freqüência de alarmes falsos se mantenha dentro de um limite tolerado, especificado pelo usuário. Este limite pode ser especificado na forma de um valor mínimo aceitável $\left(N M A_{0 \min }\right)$ para o número esperado de amostras até um alarme falso, $N M A_{0}$. O número esperado de amostras até um alarme falso é dado por

$$
N M A_{0}=1 / \alpha
$$

sendo $\alpha$ a probabilidade de alarme falso em cada amostragem, dada por:

$$
\alpha=1-\sum_{j=0}^{\lfloor L S\rfloor}\left(\begin{array}{l}
n \\
j
\end{array}\right) p_{0}^{j}\left(1-p_{0}\right)^{n-j}
$$

onde $\lfloor L S C\rfloor$ é a parte inteira do $L S C$.

Como o número de itens não conformes em cada amostra é obrigatoriamente inteiro, é boa prática adotar limites de controle fracionários, para evitar a possibilidade de o número de itens não conformes em alguma amostra coincidir com o valor de $L S C$. Isso elimina a chance de ambigüidade e possível erro do usuário na interpretação do sinal ou nãosinal. Assim, neste procedimento, adotaremos sempre $L S C$ $=\lfloor L S C\rfloor+0,5$.

Para comparação das alternativas viáveis para fins de decisão, pode-se adotar como medida de desempenho o número esperado de amostras até um alarme verdadeiro, $N M A_{1}$, dado por

$$
N M A_{1}=1 /(1-\beta)
$$

onde

$$
\beta=\sum_{j=0}^{\lfloor L S\rfloor}\left(\begin{array}{l}
n \\
j
\end{array}\right) p_{1}^{j}\left(1-p_{1}\right)^{n-j}
$$

sendo $p_{1} \mathrm{o}$ valor considerado da fração não conforme fora de controle. Nota-se, então, que $N M A_{1}$ é função de $n$, da parte inteira do $L S C$ e do valor de $p_{1}$.

Quando o número de valores aceitáveis para $n$ é pequeno, é fácil gerar todas as alternativas $(n,\lfloor L S C\rfloor)$ viáveis, por exemplo numa planilha eletrônica, e escolher a melhor com base nos valores de $N M A_{1}$ de cada alternativa para o valor (ou valores) de $p_{1}$ considerado(s). O $L S C$, porém, não é mais calculado pela fórmula (1). Para cada valor específico de $n$, deve-se usar o menor valor de $\lfloor L S C\rfloor+0,5$ que ainda garanta um $N M A_{0}$ não inferior ao valor mínimo especificado pelo usuário, $N M A_{0 \min }$. Então temos, de (3), que

$$
N M A_{0} \geq N M A_{0 \text { min }} \Rightarrow \alpha \leq \alpha_{\text {max }}=1 / N M A_{0 \text { min }}
$$

De (7) e (4) é fácil ver que, para cada valor de $n$ considerado, o valor de $\lfloor L S C\rfloor$ a adotar é o menor inteiro tal que a probabilidade binomial $\left(n, p_{0}\right)$ acumulada até ele seja ainda maior ou igual a $1-\alpha_{\max }$, sendo $\alpha_{\max }$ dado por (7). Ou seja,

$$
\lfloor L S C\rfloor=\min \left(i \in\{0,1,2, \ldots n\} \mid \sum_{j=0}^{i}\left(\begin{array}{l}
n \\
j
\end{array}\right) p_{0}^{j}\left(1-p_{0}\right)^{n-j} \geq 1-\alpha_{\max }\right)
$$

Com isso, tem-se, para cada valor de $n$ operacionalmente admissível, um $L S C$ associado. Para cada par $(n, L S C)$ assim determinado, pode-se obter com (4) e (3) os valores de $\alpha$ e $N M A_{0}$ e, em seguida, obtém-se, com (6) e (5), $\beta$ e $N M A_{1}$ para o valor ou conjunto de valores de $p_{1}$ que interesse considerar.

Até agora não entrou em consideração o intervalo de tempo entre retirada de amostras, $h$. Os valores de $N M A_{1}$ 
isoladamente não fornecem informação suficiente para a tomada de decisão sobre os parâmetros do gráfico de controle, já que o tempo esperado entre a ocorrência de uma causa especial e a sua detecção — que é o que interessa ao usuário, pois durante esse tempo o processo estará fora de controle - depende não apenas de $N M A_{1}$ mas também de $h$; além disso, não se pode esquecer dos custos das inspeções de amostras, que também dependem do valor de $h$. Resumindo, tanto os custos de amostragem como o tempo esperado de detecção de uma causa especial são função de $n$ e de $h$, de modo que a decisão sobre o valor de $n$ não pode ser dissociada da decisão sobre o valor de $h$. Examinemos agora essas duas funções, o custo de amostragem e o tempo esperado até a detecção. Supondo que o custo de retirar e inspecionar uma amostra seja proporcional ao número de itens na amostra, $n$, o custo de amostragem por unidade de tempo é proporcional ao número de itens amostrados por unidade de tempo, que é igual à razão $n / h$. Quanto ao tempo esperado até o sinal, denotado por TES (COSTA et al., 2005), ele é dado por:

$$
T E S=\left(N M A_{1}-0,5\right) h
$$

O usuário tem então duas abordagens possíveis para determinar o par $(n, h)$ a ser utilizado: (a) fixar como restrição um valor máximo admitido, $r_{m a ́ x}$, para o número médio de itens inspecionados por unidade de tempo $r=n / h$, e determinar o par $(n, h)$ que, atendendo a essa restrição, minimiza o tempo esperado até o sinal TES; ou (b) fixar como restrição um valor máximo tolerado para o TES, TES ${ }_{\max x}$, e determinar o par $(n, h)$ que minimiza o número médio de itens inspecionados por unidade de tempo $r=n / h$.

Epprecht e Santos (1998) mostraram que, na solução de qualquer um desses dois problemas, o valor ótimo de $n$ é obtido pela minimização de

$$
g=\left(N M A_{1}-0,5\right) n
$$

Esta função $g$ foi posteriormente usada por Astolfi e Hamacher (2002) para otimização do projeto de gráficos de $n p$. Quanto menor o valor de $g$, maior a eficiência do gráfico.

Com o valor de $n$ que minimiza $g$, o valor de $h$ fica automaticamente determinado pela restrição. Especificamente:

No caso de o usuário ter escolhido um valor máximo admissível, $r_{\text {maxx }}$, para a razão $n / h$, o menor valor admissível para $h$ é dado por

$$
h_{\min }=n / r_{\max }
$$

No caso de o usuário ter especificado um TES máximo tolerado, $T E S_{\text {máx }}$, para um certo $p_{1}$ de interesse, o maior valor admissível para $h$ é obtido (vide a equação (9)) por:

$$
h_{\text {max }}=T E S_{\text {max }} /\left(N M A_{1}-0,5\right)
$$

Por sua vez, esse limite $T E S_{\text {max }}$ pode ser arbitrado, mas também pode ser definido, para cada valor de $p_{1}$ considerado, em função de um valor máximo tolerado (maior que $p_{0}$ ) para a fração não conforme total esperada. A fração não conforme total resulta da combinação da produção, com fração não conforme $p_{0}$, durante o tempo em que o processo permanecer em controle com a produção, com fração não conforme $p_{1}$, durante o tempo em que o processo permanecer fora de controle. Assim, caso se conheçam o valor esperado do tempo em controle e o valor de $p_{1}$ (ou a distribuição de probabilidades dos valores de $p_{1}$ ) quando o processo sai de controle, torna-se possível relacionar a fração não conforme total com o valor de TES. Contudo, o valor esperado do tempo em controle e a distribuição dos valores de $p_{1}$ quando o processo sai de controle são, em geral, difíceis de estimar. Neste caso, pode-se ainda, mediante algumas hipóteses simplificadoras, resolver o problema para alguns cenários (obtendo, para cada cenário, um valor de $T E S_{\text {máx }}$ e um valor de $h_{\text {maxx }}$ ), e adotar a solução mais conservadora (que garanta, mesmo no pior caso, que a fração não conforme total esperada não ultrapasse o valor máximo tolerado), ou seja, adotar o menor dos valores de $h_{\text {máx }}$ assim determinados.

Por exemplo, pode-se considerar um período de tempo de duração $T$ tal que seja razoável supor desprezível a probabilidade de o processo sair de controle mais de uma vez nesse período. De acordo com essa hipótese simplificadora, durante o intervalo de duração $T$, ou o processo permanece em controle ou sai de controle apenas uma vez. Mesmo que não se conheça a distribuição do tempo em controle e nem mesmo o seu valor esperado, a equipe do processo pode se sentir confortável para definir (conservadoramente, se desejado) um intervalo nessas condições.

Então, no pior caso, durante o intervalo considerado, o processo sairá de controle apenas uma vez, e a proporção média de tempo que o processo permanece fora de controle será $T E S / T$. Ao supor que o tempo que o processo opera fora de controle é igual a TES, está-se implicitamente considerando desprezível o tempo entre a retirada da amostra e a eliminação da causa especial, o que é uma suposição razoável se o tempo para inspeção da amostra for muito menor que TES e se a produção for interrompida para a investigação da causa especial. Então, no caso de o processo sair de controle e a fração não conforme aumentar para $p_{1}$, a fração não conforme total esperada, $p c$ (onde o " $c$ " vem de "combinada"), será dada por

$p c=\left[(T-T E S) p_{0}+T E S p_{1}\right] / T$

Para um limite máximo tolerado da fração não conforme esperada, $p c_{\max }$, tem-se, de (13): 


$$
T E S_{\text {máx }}=\left[\left(p c_{\text {máx }}-p_{0}\right) /\left(p_{1}-p_{0}\right)\right] T
$$

e, de (14) e (12), segue:

$$
h_{\text {máx }}=\left[\left(p c_{\text {max }}-p_{0}\right) /\left(p_{1}-p_{0}\right)\right] T /\left(N M A_{1}-0,5\right)
$$

Assim, dados $n$ e $L S C$, obtêm-se os valores máximos admissíveis de $h$ para os valores de $p_{1}$ considerados plausíveis (lembrando que, para cada para par $(n, L S C), N M A_{1}$ é função apenas de $p_{1}$ ). Para garantir $p c \leq p c_{\text {máx }}$ mesmo no pior caso, deve-se adotar o menor dos valores de $h_{\max }$ assim determinados.

Para maior clareza, o procedimento está resumido no Apêndice, na forma de uma seqüência de passos.

\section{ANÁLISE DO CASO REAL}

O caso real que deu origem a este artigo trata do empacotamento automático de cadernos de jornais no parque gráfico de um grande jornal nacional. A unidade (item) desse processo é o pacote, que contém um número préespecificado, $N$, de cadernos ( $N$ pode variar de 20 a 400 , dependendo do número de páginas do caderno; $N=50$ é um valor típico). O item é classificado como não conforme sempre que possuir um número de cadernos diferente de $N$. As especificações são de que no máximo $0,5 \%$ dos pacotes tenham um número incorreto de cadernos. Assim, $p_{0}=0,005$ (na verdade, essa é a fração não conforme aceitável, estipulada; o processo tem capacidade para $p_{0}<0,005$ e, em controle, gera realmente menos de $0,5 \%$ de pacotes não conformes. A empresa, contudo, não dispõe de uma estimativa precisa da fração não conforme real, e adota $p_{0}=0,005$ por definição). Os cadernos são empacotados por seis máquinas em paralelo.

A Gerência Industrial desejava estabelecer um plano de amostragem para monitoramento do processo: tamanho de amostra, limites de controle e intervalo de tempo entre retirada de amostras. O controle deve ser feito para cada máquina, independentemente.
A inspeção de cada item (pacote) das amostras é manual: um inspetor conta o número de cadernos; dado o número de cadernos por pacote, a inspeção de cada pacote é demorada. Acresça-se a isso o fato de haver seis máquinas a serem monitoradas, e a alta taxa de produção, que, impondo intervalos entre amostras não muito longos, restringe o tempo para inspeção de uma amostra e, em decorrência, o tamanho das amostras. Tanto a taxa de produção como o número de cadernos por pacote dependem do número de páginas por caderno e da velocidade de rotação das máquinas, mas valores típicos estão na faixa de 35.000 a 50.000 cadernos por hora, o que corresponde a 700 a 1.000 pacotes por hora. Portanto, a amostragem precisa ser freqüente. Em suma, não se admitem tamanhos de amostra ( $n$ ) da ordem de centenas de unidades (pacotes). Embora a Gerência Industrial da empresa não tivesse uma definição precisa sobre o que seria um tamanho de amostra máximo admissível — estando claro que isso dependeria do intervalo de tempo entre retiradas de amostras -, ela afirmou que $n>20$ seria inadmissível em qualquer caso.

Antes de aplicar o procedimento proposto, consideremos, a título de ilustração, o uso dos limites de três sigma com amostras pequenas: $n=5,10,15$ e 20 . A Tabela 1 fornece a linha média $\left(L M=n p_{0}\right), \mathrm{o} L S C$, o número médio de amostras até um alarme falso $\left(N M A_{0}\right.$, calculado por (3) e (4)) e o número médio de amostras até um alarme verdadeiro $\left(N M A_{1}\right.$, calculado por (5) e (6)) para $p_{1}=0,01,0,02$ e 0,04 .

A inadequação dos limites de três sigma pode ser vista pelos valores de $N M A_{0}$, da ordem de um alarme falso em média a cada 40,4, 20,5 ou mesmo 13,8 amostras, para $n=5,10$ e 15. Já para $n=20, \mathrm{o} N M A_{0}$ assume um valor mais razoável (em média um alarme falso a cada 223,5 amostras); porém, o número médio de amostras para se detectar um aumento na fração defeituosa do processo é grande.

Isso confirma e ilustra a inaplicabilidade do procedimento usual de planejamento de um gráfico de controle (escolher um tamanho de amostra e calcular os limites de três sigma) nesses casos de $n p_{0}$ pequeno. Foi por isso aplicado o procedimento proposto neste artigo, conforme será descrito a seguir.

Tabela 1: Propriedades dos gráficos de controle $n p$ com limites de três sigma ( $n$ pequeno e $p_{0}=0,005$ ).

\begin{tabular}{ccccccc}
\hline & & & & \multicolumn{3}{c}{ NMA, para $_{\mathbf{1}}$ igual a ... } \\
\cline { 5 - 7 }$n$ & LM & LSC & NMA $_{\mathbf{0}}$ & $\mathbf{0 , 0 1}$ & $\mathbf{0 , 0 2}$ & $\mathbf{0 , 0 4}$ \\
\hline 5 & 0,025 & 0,50 & 40,4 & 20,40 & 10,41 & 5,42 \\
10 & 0,050 & 0,72 & 20,5 & 10,06 & 5,47 & 2,98 \\
15 & 0,075 & 0,89 & 13,8 & 7,15 & 3,83 & 2,18 \\
20 & 0,100 & 1,05 & 223,5 & 59,31 & 16,69 & 5,27 \\
\hline
\end{tabular}




\section{APLICAC̄̃̃O DO PROCEDIMENTO PROPOSTO}

A descrição da aplicação do procedimento proposto ao planejamento do gráfico de controle do processo de empacotamento automático de cadernos de jornais será feita aqui seguindo os passos descritos no Apêndice.

\section{Passo 1 - Fixar um limite máximo para a probabilidade} de alarme falso:

Adotou-se inicialmente $N M A_{\text {0min }}=100 \Rightarrow \alpha_{\text {máx }}=1 / 100$ $=0,01$ (Passo 1). Escolheu-se este valor para o $N M A_{0 \text { min }}$ porque já se tinha visto, em análise prévia, que $N M A_{0 \min }=$ 200 levaria a grandes valores de $N M A_{1}$; assim, considerou-se $N M A_{\text {0min }}=100$ uma solução de compromisso aceitável.

Passo 2 - Determinar, para cada valor de $n$ admissivel, o menor $\lfloor L S C\rfloor$ que não viole essa restrição:

Lembrando que, embora a Gerência Industrial da empresa dissesse que o tamanho de amostra máximo admissível dependeria do intervalo de tempo entre retiradas de amostras, ela deixou claro que $n>20$ seria inadmissível em qualquer caso, a análise foi feita para $n$ na faixa de variação de 2 até 20 . Foi utilizada uma planilha eletrônica para os cálculos (no caso, o Microsoft Excel). Os valores de $n$ foram dispostos na primeira coluna da planilha, e $\lfloor L S C\rfloor$ foi calculado na segunda coluna (pela função CRIT. BINOM, descrita no Apêndice). Calculou-se também, a seguir, para cada par $(n,\lfloor L S C\rfloor)$, o valor de $N M A_{0}$ pelas fórmulas (4) e (3).

Passo 3 - Calcular, para cada par ( $n,\lfloor L S C\rfloor)$, o número médio de amostras até o sinal $\left(N M A_{1}\right)$ para um conjunto de valores da fração defeituosa fora de controle:

Para cada par $(n,\lfloor L S C\rfloor)$, calculou-se o $N M A_{1}$ pelas fórmulas (6) e (5), para $p_{1}=0,01,0,02$ e 0,04 .

A Tabela 2 apresenta a planilha resultante.

Comentários: Os $N M A_{1}$ s obtidos (vide Tabela 2) foram considerados muito grandes. A baixa eficiência do esquema de controle aqui é inevitável: ela advém do $p_{0}$ muito pequeno do processo, que requereria grandes tamanhos de amostra para um controle eficiente; porém a freqüência de amostragem, que precisa ser alta (dada a alta taxa de produção), e o tempo necessário para inspecionar cada pacote impedem a retirada de grandes amostras. Dado esse conflito entre as limitações operacionais e os tamanhos de amostra que seriam necessários para um controle eficiente, o melhor que pode ser feito é selecionar a solução preferível: não a ideal, mas a melhor possível.
Na tentativa de encontrar uma melhor relação entre ocorrências de alarmes falsos e rapidez de detecção, voltou-se ao Passo 1: reduziu-se $N M A_{0 \min }$ em um terço, para 67 amostras, o que equivale a $\alpha_{\text {max }}=0,014925$, e repetiram-se os passos 2 e 3. Este valor para $N M A_{0 \min }$ pode parecer muito pequeno, mas o $N M A_{0}$ real será maior, e mesmo sempre maior que o $N M A_{0}$ calculado para $p_{0}=0,005$, já que que a fração não conforme em controle do processo é na realidade menor que o valor nominal 0,005 . A nova planilha está na Tabela 3 .

Comparando os LSCs Tabela 3 com os da Tabela 2, observa-se que o único projeto que se alterou com o novo valor para $\alpha_{\max }$ foi o projeto para $n=3$. Os menores valores de $N M A_{1}$ são obtidos, para $p_{1}=0,01, \operatorname{com} n=3$; para $p_{1}=0,02$, com $n=20\left(N M A_{1}=16,9\right)$, mas seguido de perto pelo valor de $N M A_{1}=17,0$, para $n=3$; e, para $p_{1}=0,04$, os valores de $N M A_{1}$ menores que com $n=3$ ocorrem apenas com $n \geq 15$. Este resultado pode parecer contradizer uma "verdade" estatística, de que o poder dos gráficos de controle (ou dos testes de hipótese de maneira geral) aumenta com o tamanho de amostra. A razão, porém, é fácil de ver: para $n=2 \mathrm{e}$ $n=3,\lfloor L S C\rfloor=0$, de modo que um pacote não conforme na amostra já é considerado sinal de descontrole; já para $n \geq 4$, $\lfloor L S C\rfloor=1$ (pois $\lfloor L S C\rfloor=0$ geraria um $N M A_{0}$ inaceitável). Este aumento no $L S C$, requerendo dois pacotes não conformes para o sinal, reduz demasiadamente o poder do gráfico. Este problema - que não surge com gráficos de controle por variáveis - é inerente ao CEP por atributos, e deve-se ao caráter discreto da estatística amostral e às descontinuidades da sua distribuição acumulada, que torna impossível manter uma probabilidade de alarme falso constante para diferentes tamanhos de amostra. Assim, não há paradoxo em que gráficos com menor tamanho de amostra apresentem maior poder: está-se comparando testes de hipótese com diferentes níveis de significância.

A Figura 1, que apresenta o gráfico de $N M A_{1} \times n$, mostra esse efeito. O grande "salto" visível ocorre exatamente de $n=3$ para $n=4$, coincidindo com a mudança de $\lfloor L S C\rfloor$ de 0 para 1.

Passo 4 - Cálculo da função g e identificação do par (n, $\lfloor L S C\rfloor)$ ótimo:

A Tabela 4 é igual à Tabela 3 com o acréscimo de três colunas, que apresentam os valores da função $g$ para os diversos pares $(n,\lfloor L S C\rfloor)$. O mínimo desta função, para qualquer dos valores de $p_{1}$, ocorre para $n=2$, sendo o valor para $n=3$ também muito próximo do mínimo.

A função $g$ mostra que, embora $n=20$ forneça menores $N M A_{1}$ s para $p_{1}=0,02$ e $p_{1}=0,04$ do que $n=2$ ou $n=3$, se com amostras menores se puder adotar intervalos entre amostras proporcionalmente menores (por exemplo, $\operatorname{com} n=2$, adotarse-ia um intervalo de tempo entre amostras 10 vezes mais 
curto que com $n=20$ ), então com $n=2$ ou $n=3$ o TES será muito menor que com $n=20$. Isso porque, como foi dito, se pré-fixarmos uma taxa de amostragem $n / h=r_{\text {max }}$, fazendo $h$ proporcional a $n$, então o TES será proporcional ao valor da função $g$. Portanto, examinando os valores da função $g$, as melhores soluções são as soluções com $n=2$ e $n=3$. A semelhança dos valores da função $g$ para essas duas soluções mostra que, em termos de detecção, elas não diferem significativamente: se o intervalo de tempo entre amostras de tamanho 2 for igual a $2 / 3$ do intervalo de tempo entre amostras de tamanho 3, os tempos médios até a sinalização serão próximos. A escolha final torna-se uma questão de preferência subjetiva, de razões operacionais, psicológicas ou outras, e fica a critério do usuário.
Em qualquer dessas duas opções, $L S C=0+0,5=0,5$; um pacote não conforme é sinal de alteração no processo.

\section{Passo 5 - Escolha do valor de $h$ :}

Falta escolher o intervalo de tempo entre amostras a utilizar, seja em função de um valor máximo aceitável $r_{\text {maxx }}$ para a taxa de amostragem $r=n / h$, seja em função do maior TES tolerado, $T E S_{\text {max }}$ (que ainda pode ser determinado em função do valor máximo aceitável para a fração não conforme total esperada $\left.p_{\text {cmáx }}\right)$.

A alternativa adotada foi escolher um limite máximo para o TES, TES ${ }_{\text {máx }}$. A decisão decorreu da observação dos valores de $N M A_{1}$, ainda grandes nos melhores casos, tornando

Tabela 2: Planilha de auxílio ao planejamento do gráfico de controle do empacotamento automático de cadernos de jornais $\left(p_{0}=0,005\right.$ e $\left.N M A_{\text {omin }}=100\right)$.

\begin{tabular}{|c|c|c|c|c|c|}
\hline \multirow[b]{2}{*}{$\mathbf{n}$} & \multirow{2}{*}{$\begin{array}{c}\text { PARTE } \\
\text { INTEIRA DO LSC }\end{array}$} & \multirow{2}{*}{$\mathbf{N M A}_{0}$} & \multicolumn{3}{|c|}{ NMA, para $p_{1}$ igual a: } \\
\hline & & & 0,01 & 0,02 & 0,04 \\
\hline 2 & 0 & 100,3 & 50,3 & 25,3 & 12,8 \\
\hline 3 & 1 & 13377,9 & 3355,7 & 844,6 & 214,0 \\
\hline 4 & 1 & 6711,3 & 1689,1 & 428,0 & 109,9 \\
\hline 5 & 1 & 4040,3 & 1020,3 & 260,3 & 67,8 \\
\hline 6 & 1 & 2702,5 & 684,7 & 175,8 & 46,4 \\
\hline 7 & 1 & 1936,8 & 492,4 & 127,3 & 34,0 \\
\hline 8 & 1 & 1457,4 & 371,7 & 96,7 & 26,2 \\
\hline 9 & 1 & 1137,4 & 291,1 & 76,2 & 20,9 \\
\hline 10 & 1 & 912,9 & 234,4 & 61,8 & 17,2 \\
\hline 11 & 1 & 749,4 & 193,1 & 51,2 & 14,4 \\
\hline 12 & 1 & 626,6 & 162,0 & 43,3 & 12,4 \\
\hline 13 & 1 & 532,0 & 138,0 & 37,1 & 10,7 \\
\hline 14 & 1 & 457,5 & 119,0 & 32,2 & 9,4 \\
\hline 15 & 1 & 397,8 & 103,8 & 28,3 & 8,4 \\
\hline 16 & 1 & 349,2 & 91,5 & 25,1 & 7,5 \\
\hline 17 & 1 & 309,2 & 81,2 & 22,4 & 6,8 \\
\hline 18 & 1 & 275,7 & 72,7 & 20,2 & 6,2 \\
\hline 19 & 1 & 247,5 & 65,5 & 18,3 & 5,7 \\
\hline 20 & 1 & 223,5 & 59,3 & 16,7 & 5,3 \\
\hline
\end{tabular}


evidente a necessidade de adotar intervalos de tempo entre amostras que pudessem garantir ainda assim tempos de sinalização razoáveis. Por sua vez, definiu-se $T E S_{\text {máx }}$ em função do valor máximo tolerado para a fração não conforme total esperada. No caso analisado, os tempos foram medidos em número de itens (pacotes), e foi adotado $T=800$ pacotes. Foi especificado o valor 0,011 como $p_{\text {cmáx }}$ para cada máquina. Este valor veio de mais uma consideração simplificadora: de que se apenas uma máquina sair de controle e sua fração não conforme total no intervalo de tempo $T$ for $p_{\text {cmáx }}$, a fração não conforme da produção total das seis máquinas será $p_{\text {total }}=\left[(5 / 6) p_{0}+(1 / 6) p_{\text {cmáx }}\right]$ o que $\left(\operatorname{com} p_{0}=0,005 \mathrm{e}\right.$ $\left.p_{\text {cmax }}=0,011\right)$ é igual a apenas 0,006 . É imediato obter $p_{\text {cmáx }}$ a partir de qualquer outro limite estipulado para a fração não conforme da produção total das seis máquinas.
A Tabela 5 apresenta, para $n=2$ e $n=3$, os valores de $h_{\max }$ calculados, pela equação (15), em função deste valor de $p_{\text {cmáx }}$ e de $p_{1}$ (para $p_{1}=0,01,0,02$ e 0,04 ). Para fornecer proteção no pior caso, $n=2$ requer $h \leq 11$ pacotes, e $n=3$ requer $h \leq 16$ pacotes. Portanto, deverão ser inspecionados dois pacotes a cada 11 pacotes produzidos ou três pacotes a cada 16 pacotes produzidos. Um pacote não conforme será sinal de descontrole, e a máquina deve ser parada e regulada. Note que neste caso não faz sentido manter um gráfico de controle, pois os valores registrados seriam seqüências de zeros, com algum ocasional "1", que já seria um sinal (sendo muito improvável um valor maior do que "1"). A única informação veiculada por tal gráfico seria o número de amostras entre dois sinais.

Essa taxa de inspeção (2 pacotes a cada 11 pacotes

Tabela 3: Planilha de auxílio ao planejamento do gráfico de controle do empacotamento automático de cadernos de jornais $\left(p_{0}=0,005\right.$ e $\left.N M A_{0 \text { min }}=67\right)$.

\begin{tabular}{|c|c|c|c|c|c|}
\hline \multirow[b]{2}{*}{$n$} & \multirow{2}{*}{$\begin{array}{l}\text { PARTE } \\
\text { INTEIRA DO } \\
\text { LSC }\end{array}$} & \multirow[b]{2}{*}{$\mathrm{NMA}_{0}$} & \multicolumn{3}{|c|}{ NMA, para $p_{1}$ igual a: } \\
\hline & & & 0,01 & 0,02 & 0,04 \\
\hline 2 & 0 & 100,3 & 50,3 & 25,3 & 12,8 \\
\hline 3 & 0 & 67,0 & 33,7 & 17,0 & 8,7 \\
\hline 4 & 1 & 6711,3 & 1689,1 & 428,0 & 109,9 \\
\hline 5 & 1 & 4040,3 & 1020,3 & 260,3 & 67,8 \\
\hline 6 & 1 & 2702,5 & 684,7 & 175,8 & 46,4 \\
\hline 7 & 1 & 1936,8 & 492,4 & 127,3 & 34,0 \\
\hline 8 & 1 & 1457,4 & 371,7 & 96,7 & 26,2 \\
\hline 9 & 1 & 1137,4 & 291,1 & 76,2 & 20,9 \\
\hline 10 & 1 & 912,9 & 234,4 & 61,8 & 17,2 \\
\hline 11 & 1 & 749,4 & 193,1 & 51,2 & 14,4 \\
\hline 12 & 1 & 626,6 & 162,0 & 43,3 & 12,4 \\
\hline 13 & 1 & 532,0 & 138,0 & 37,1 & 10,7 \\
\hline 14 & 1 & 457,5 & 119,0 & 32,2 & 9,4 \\
\hline 15 & 1 & 397,8 & 103,8 & 28,3 & 8,4 \\
\hline 16 & 1 & 349,2 & 91,5 & 25,1 & 7,5 \\
\hline 17 & 1 & 309,2 & 81,2 & 22,4 & 6,8 \\
\hline 18 & 1 & 275,7 & 72,7 & 20,2 & 6,2 \\
\hline 19 & 1 & 247,5 & 65,5 & 18,3 & 5,7 \\
\hline 20 & 1 & 223,5 & 59,3 & 16,7 & 5,3 \\
\hline
\end{tabular}


produzidos ou 3 pacotes a cada 16 pacotes produzidos) é muito alta. Teoricamente, uma possibilidade seria colocar inspetores em paralelo. Outra alternativa seria alterar a especificação de $p_{\text {cmáx }}$. Por exemplo, com $p_{\text {total }}=0,008$, ter-se-ia $p_{\text {cmáx }}=0,023 \mathrm{e}$, por (15), os valores de $h_{\text {máx }}$ seriam 3 vezes maiores que os apresentados na Tabela 5. Com isso, poder-se-ia inspecionar apenas 2 pacotes a cada 33 pacotes produzidos ou 3 pacotes a cada 50 pacotes produzidos.

\section{CONCLUSÕES}

No controle estatístico de processos pelo gráfico de $n p$ (ou de $p$ ), quando a fração defeituosa em controle, $p_{0}$, é muito pequena e razões de ordem prática, operacional ou econômica impedem a retirada de grandes amostras, os tradicionais limites de três sigma podem levar a freqüências de alarmes falsos inaceitavelmente altas. Mais ainda, para vários tamanhos de amostra dentro dessa faixa em que $n p_{0}$ é muito pequeno, não existe nenhum valor para o limite superior de controle que proporcione um bom desempenho para o gráfico: com alguns valores, a taxa de alarmes falsos é inaceitavelmente alta, e com valores maiores, o poder do gráfico em sinalizar descontroles é inaceitavelmente baixo.
Isso advém das grandes descontinuidades da distribuição acumulada do número de defeituosos na amostra, e faz com que os tamanhos de amostra que fornecem desempenho satisfatório para o gráfico formem um conjunto esparso, de valores não consecutivos.

Nestes casos, a escolha do tamanho de amostra (n) e do limite de controle ( $L S C$ ) não pode ser feita em duas etapas, primeiro escolhendo $n$ e em seguida calculando o valor de $L S C$ por uma fórmula: o par $(n, L S C)$ precisa ser escolhido conjuntamente, calculando e comparando as medidas de desempenho das diversas alternativas, como da maneira mostrada neste artigo.

O esquema de controle de $n p$ não é eficiente para pequenos valores de $n p_{0}$. Nessas situações em que $p_{0}$ é pequeno e não são viáveis grandes tamanhos de amostra, o usuário não poderá dispor de um procedimento estatístico que ofereça ao mesmo tempo grande proteção contra alarmes falsos e rápida detecção de aumentos em $p_{0}$; ele terá que conviver, inevitavelmente, com probabilidades de alarme falso maiores do que as usualmente consideradas razoáveis (valores como 0,0027 e mesmo 0,0050 podem não ser possíveis), e/ou com um gráfico de controle de limitada capacidade de sinalização. Isso, mesmo escolhendo o "melhor" par $(n, L S C)$.

Figura 1: Variação do NMA 1 com o tamanho de amostra (com a restrição $\alpha \leq 0,014925$ ).

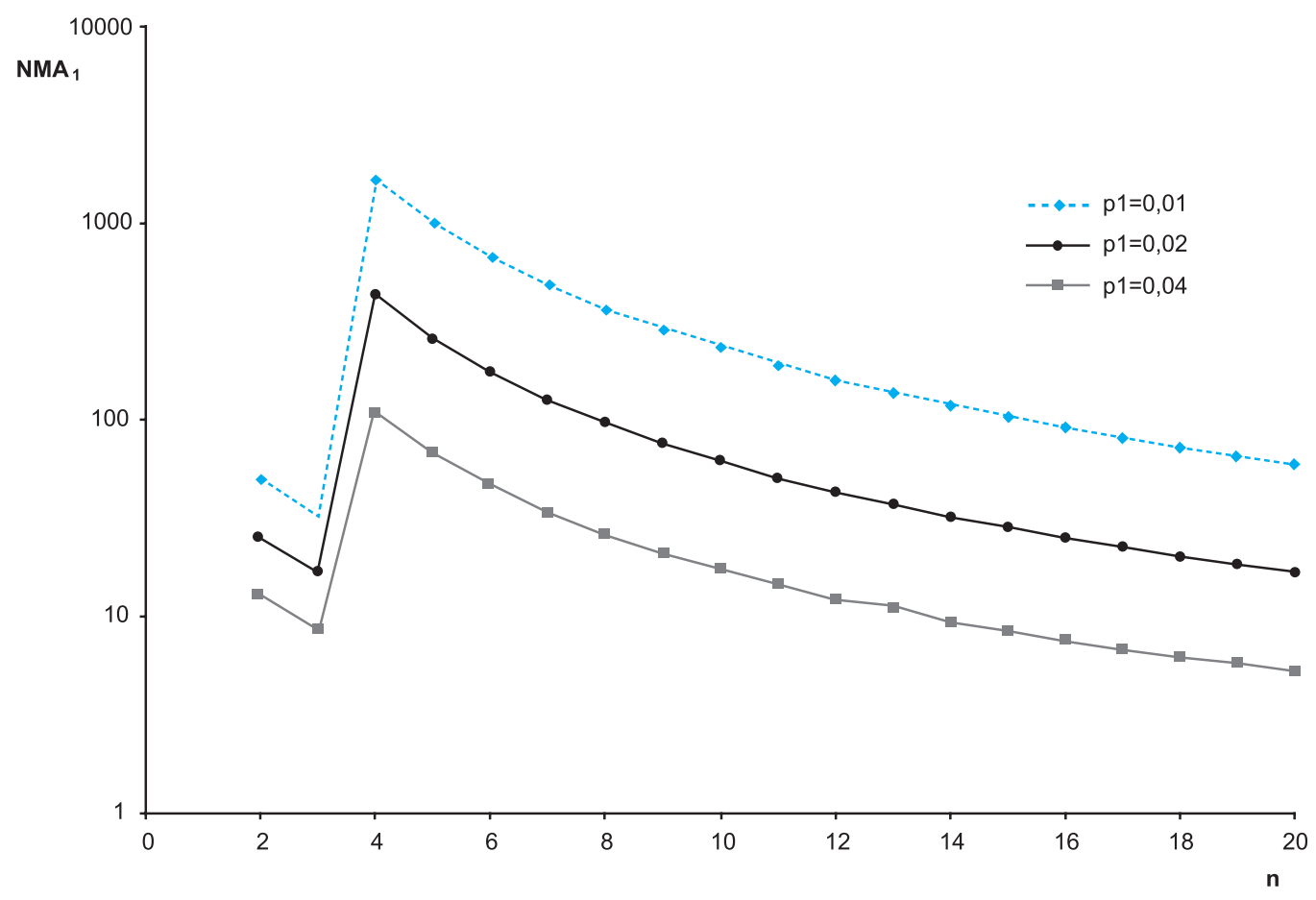


Tabela 4: Planilha de auxílio ao planejamento do gráfico de controle do empacotamento automático de cadernos de jornais $\left(p_{0}=0,005\right.$ e $\left.\mathbf{N M A _ { 0 \operatorname { m i n } }}=67\right)$, com acréscimo da função $\mathbf{g}$.

\begin{tabular}{|c|c|c|c|c|c|c|c|c|}
\hline \multirow{2}{*}{$n$} & \multirow{2}{*}{$\begin{array}{c}\text { PARTE } \\
\text { INTEIRA } \\
\text { DO LSC }\end{array}$} & \multirow{2}{*}{$N M A_{0}$} & \multicolumn{3}{|c|}{ NMA, para $p_{1}$ igual a: } & \multicolumn{3}{|c|}{ FUNÇÃO g para $p_{1}$ igual a: } \\
\hline & & & 0,01 & 0,02 & 0,04 & 0,01 & 0,02 & 0,04 \\
\hline 2 & 0 & 100,3 & 50,3 & 25,3 & 12,8 & 99,50 & 49,51 & 24,51 \\
\hline 3 & 0 & 67,0 & 33,7 & 17,0 & 8,7 & 99,51 & 49,51 & 24,53 \\
\hline 4 & 1 & 6711,3 & 1689,1 & 428,0 & 109,9 & 6754,41 & 1709,98 & 437,77 \\
\hline 5 & 1 & 4040,3 & 1020,3 & 260,3 & 67,8 & 5098,76 & 1298,77 & 336,30 \\
\hline 6 & 1 & 2702,5 & 684,7 & 175,8 & 46,4 & 4105,33 & 1052,01 & 275,39 \\
\hline 7 & 1 & 1936,8 & 492,4 & 127,3 & 34,0 & 3443,01 & 887,48 & 234,75 \\
\hline 8 & 1 & 1457,4 & 371,7 & 96,7 & 26,2 & 2969,89 & 769,93 & 205,71 \\
\hline 9 & 1 & 1137,4 & 291,1 & 76,2 & 20,9 & 2615,03 & 681,74 & 183,92 \\
\hline 10 & 1 & 912,9 & 234,4 & 61,8 & 17,2 & 2339,01 & 613,14 & 166,96 \\
\hline 11 & 1 & 749,4 & 193,1 & 51,2 & 14,4 & 2118,17 & 558,24 & 153,38 \\
\hline 12 & 1 & 626,6 & 162,0 & 43,3 & 12,4 & 1937,47 & 513,31 & 142,27 \\
\hline 13 & 1 & 532,0 & 138,0 & 37,1 & 10,7 & 1786,86 & 475,85 & 133,00 \\
\hline 14 & 1 & 457,5 & 119,0 & 32,2 & 9,4 & 1659,42 & 444,15 & 125,16 \\
\hline 15 & 1 & 397,8 & 103,8 & 28,3 & 8,4 & 1550,17 & 416,97 & 118,43 \\
\hline 16 & 1 & 349,2 & 91,5 & 25,1 & 7,5 & 1455,47 & 393,40 & 112,61 \\
\hline 17 & 1 & 309,2 & 81,2 & 22,4 & 6,8 & 1372,60 & 372,78 & 107,51 \\
\hline 18 & 1 & 275,7 & 72,7 & 20,2 & 6,2 & 1299,48 & 354,57 & 103,01 \\
\hline 19 & 1 & 247,5 & 65,5 & 18,3 & 5,7 & 1234,46 & 338,38 & 99,02 \\
\hline 20 & 1 & 223,5 & 59,3 & 16,7 & 5,3 & 1176,29 & 323,90 & 95,45 \\
\hline
\end{tabular}

Tabela 5: $\boldsymbol{h}$ máximo em função de $\boldsymbol{n}$ e $\boldsymbol{p}_{1}$, para $\boldsymbol{p}_{\text {cmáx }}=0,011$ e $\boldsymbol{T}=800$ pacotes $\left(p_{0}=0,005\right.$ e $\left.N M A_{0 \text { min }}=67\right)$.

\begin{tabular}{c|ccc|ccc}
\hline & \multicolumn{3}{|c|}{ NMA $_{\mathbf{1}}$ para $\mathbf{p}_{\mathbf{1}}$ igual a... } & \multicolumn{3}{c}{$\mathbf{h}_{\text {máx }}$ para $\mathbf{p}_{\mathbf{1}}$ igual a... } \\
$n$ & 0,01 & 0,02 & 0,04 & 0,01 & 0,02 & 0,04 \\
\hline 2 & 50,25 & 25,25 & 12,76 & 19,3 & 12,9 & 11,2 \\
3 & 33,67 & 17,00 & 8,68 & 28,9 & 19,4 & 16,8 \\
\hline
\end{tabular}


Outra peculiaridade desses casos é que os valores extremamente baixos de $n$ e $L S C$ quase sempre tornarão sem sentido o lançamento dos dados em um gráfico. No caso real analisado, os valores registrados seriam seqüências de zeros: um valor "1" já seria um sinal. Esses baixos valores para o número de defeituosos na amostra são um obstáculo também à utilização de esquemas de controle que pretendem ser mais eficientes que o esquema clássico dos gráficos de Shewhart, como os esquemas EWMA (GAN, 1990; RODRIGUES, 2005, Cap. 5), CuSum (GAN, 1993; Rodrigues, 2005, Cap. 5) ou os gráficos adaptativos (de parâmetros variáveis: VAUGHAN, 1993; EPPRECHT e COSTA, 2001; EPPRECHT et al., 2003). Daí a importância de se escolher o melhor projeto para o esquema de controle, pelo procedimento aqui apresentado.

\section{APÊNDICE}

\section{Resumo do procedimento para o planejamento do gráfico de controle $n p$ para processos com baixa ocorrência} de itens não conformes e restritos a amostras pequenas

1. Fixar um limite máximo para a probabilidade de alarme falso $\alpha_{\text {máx }}$ (ou fixar um limite mínimo para o $N M A_{0}, N M A_{0 \text { min }}$, e calcular $\alpha_{\text {máx }}=1 / N M A_{0 \text { min }}$ );

2. Determinar, para cada valor de $n$ admissível, o menor $\lfloor L S C\rfloor$ que não viole a restrição $\alpha \leq \alpha_{\text {máx }}$, sendo $\alpha$ dado pela equação (4); ou seja, determinar $\lfloor L S C\rfloor$ pela equação $(8) ;{ }^{(*)}$

3. Calcular, para cada par $(n,\lfloor L S C\rfloor)$ obtido, o número médio de amostras até o sinal $\left(N M A_{1}\right)$, para um valor de interesse (ou conjunto de valores) da fração defeituosa fora de controle (usar as equações (5) e (6)).

4. Calcular, para cada par $(n,\lfloor L S C\rfloor)$ obtido, o valor da função $g$, pela equação (10). O par $(n,\lfloor L S C\rfloor)$ que minimiza $g$ é o par ótimo. Fazer $L S C=\lfloor L S C\rfloor+0,5$.

5. Escolha do valor de $h$ : Quando se fixa $r=r_{\text {max }}$ o intervalo de tempo entre amostras $h$ é dado por $h_{\min }=n / r_{\max }$ ), e, quando se fixa TES $=T E S_{\max x}$, é dado por $h_{\text {máx }}=T E S_{\text {max }} /\left(N M A_{1}-0,5\right)$. Neste último caso, o valor de TESmax pode ainda ser arbitrado diretamente, ou ser determinado em função de um valor máximo aceitável para a fração não conforme total, $p_{\text {cmax }}$, pela equação (14).

${ }^{(*)}$ Dada a difusão generalizada do software Microsoft Excel, é útil comentar que a equação (8) é resolvida com este software pela função CRIT.BINOM, que, tendo como parâmetros de entrada (nesta ordem) o tamanho de amostra $n$ (chamado de "Tentativas" pelo Excel), a probabilidade de sucesso $p$ (chamada de "Probabilidade_s"), e uma probabilidade acumulada (chamada de "Alfa"), fornece o menor valor inteiro, $i$, tal que a probabilidade $\operatorname{binomial}(n, p)$ acumulada até $i$ é maior ou igual a Alfa (note que, portanto, na nomenclatura do Excel, "Alfa" corresponde à probabilidade que chamamos de $1-\alpha$ ). Os parâmetros de entrada devem ser, então: "Tentativas": $n$; "Probabilidade_s": $p_{0}$; “Alfa": $\left(1-\alpha_{\max }\right)$; e a saída (valor retornado pela função) corresponde a $\lfloor L S C\rfloor$. 


\section{- Referências}

ASTOLFI, C. N. N.; HAMACHER, F. C. Projeto Ótimo de Gráficos de Controle de Processo por Atributos. Anais do XXXIV Simpósio Brasileiro de Pesquisa Operacional, Rio de Janeiro, 2002.

COSTA, A. F. B. Gráficos de Controle $\bar{X}$ para Processos Robustos. Gestão $\mathcal{E}$ Produção, v. 5, n. 3, p. 259-271, 1998

COSTA, A. F. B.; EPPRECHT, E. K.; CARPINETTI, L. C. R. Controle Estatístico de Qualidade. São Paulo: Atlas, $2^{\mathbf{a}}$ edição, 2005. 336 p.

EPPRECHT, E. K.; COSTA, A. F. B. Adaptive Sample Size Control Charts for Attributes. Quality Engineering, v. 13, n. 3, p. 465-473, 2001
EPPRECHT, E. K.; COSTA, A. F. B.; MENDES, F. C. T. Adaptive Control Charts for Attributes. IIE Transactions, v. 35, n. 6, 567-582, 2003.

EPPRECHT, E. K.; SANTOS, A. B. Um Método Simples para o Projeto Ótimo de Gráficos de $\bar{X}$. Gestão \& Produção, v. 5, n. 3, p. 206-220, 1998

GAN, F. F. Monitoring Observations Generated from a Binomial Distribution Using a Modified Exponentially Weighted Moving Average Control Chart. Journal of Statistical Computation and Simulation, v. 19, n. 1, p. 103-124, 1990.
GAN, F. F. Optimal Design of Cusum Control Charts for Binomial Counts. Journal of Applied Statistics, v. 20, n. 4, p. 445-460, 1993.

MICHEL, R.; FOGLIATTO, F. S. Projeto Econômico de Cartas Adaptativas para Monitoramento de Processos. Gestão $\&$ Produção, v. 9, n. 1, p. 17-31

MINGOTI, S. A.; FIDELIS, M. T., 2001 Aplicando a Geoestatística no Controle Estatístico de Processos. Produto $\&$ Produção, v. 5, n. 2, p. 55-70, 2002.

RODRIGUES, A. A. DE A. Gráficos de Controle por Atributos com Amostragem Dupla. Tese de Doutorado, Departamento de Engenharia Industrial, PUC-Rio, RJ, 2005

TURNES, O.; HO, L. L.; IMAÑA, C. R. Planejamento Econômico de Gráficos de $\bar{X}$ e $R$ para Processos Regenerativos e Não Regenerativos. Gestão \& Produção, v. 11 , n. 1 , p. $91-100,2004$

VAUGHAN, T. S. Variable Sampling Interval $n p$ Process Control Chart. Communications in Statistics - Theory and Methods, 22, p. 147-167, 1993.

WHEELER, D. J. Advanced Topics in Statistical Process Control - The Power of Shewhart's Charts. Knoxville, Tennessee: SPC Press, 1995. 484 p.

\section{- Agradecimentos}

Os autores agradecem o apoio do CNPq, a autorização da empresa analisada para divulgação deste trabalho e a colaboração da sua Gerência Industrial.

\section{- Sobre os autores}

\section{Adriana Leiras}

Pontifícia Universidade Católica do Rio de Janeiro (PUC-Rio)

Doutoranda do curso de Engenharia de Produção (Departamento de Engenharia Industrial)

End.: Rua Marquês de São Vicente, 225, sala 950 - 22453-900 - Rio de Janeiro - RJ

Tel./Fax: (21) 3527-1284 e (21) 3527-1288

E-mail: aleiras@ind.puc-rio.br

\section{Antonio Fernando Branco Costa}

UNESP - Faculdade de Engenharia - Campus de Guaratinguetá

Professor do Departamento de Produção

End.: Av. Ariberto Pereira da Cunha 333 - 12516-410 - Guaratinguetá - SP

Tel./Fax.: (12) 3123-2855

E-mail: fbranco@feg.unesp.br

\section{Eugenio Kahn Epprecht}

Pontifícia Universidade Católica do Rio de Janeiro (PUC-Rio)

Professor do Departamento de Engenharia Industrial

End.: Rua Marquês de São Vicente, 225, sala 950 - 22453-900 - Rio de Janeiro - RJ

Tel./Fax: (21) 3527-1284 e (21) 3527-1288

E-mail: eke@ind.puc-rio.br 